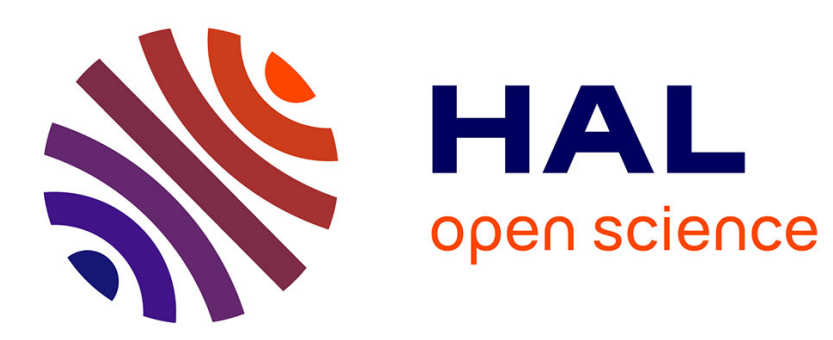

\title{
Determination of optical properties of percolated nanostructures using an optical resonator system
}

\author{
J. Sukmanowski, Y. Battie, F.X. Royer, A. En Naciri
}

\section{To cite this version:}

J. Sukmanowski, Y. Battie, F.X. Royer, A. En Naciri. Determination of optical properties of percolated nanostructures using an optical resonator system. Journal of Applied Physics, 2012, 112 (10), pp.103536. 10.1063/1.4768202 . hal-02899512

\section{HAL Id: hal-02899512 \\ https://hal.univ-lorraine.fr/hal-02899512}

Submitted on 28 Jan 2022

HAL is a multi-disciplinary open access archive for the deposit and dissemination of scientific research documents, whether they are published or not. The documents may come from teaching and research institutions in France or abroad, or from public or private research centers.
L'archive ouverte pluridisciplinaire HAL, est destinée au dépôt et à la diffusion de documents scientifiques de niveau recherche, publiés ou non, émanant des établissements d'enseignement et de recherche français ou étrangers, des laboratoires publics ou privés. 


\section{AIP Applied Physics}

\section{Determination of optical properties of percolated nanostructures using an optical resonator system}

J. Sukmanowski, Y. Battie, F. X. Royer, and A. En Naciri

Citation: J. Appl. Phys. 112, 103536 (2012); doi: 10.1063/1.4768202

View online: http://dx.doi.org/10.1063/1.4768202

View Table of Contents: http://jap.aip.org/resource/1/JAPIAU/v112/i10

Published by the American Institute of Physics.

\section{Related Articles}

Near-field simulation of obliquely deposited surface-enhanced Raman scattering substrates J. Appl. Phys. 112, 113111 (2012)

Characterization of Ru thin-film conductivity upon atomic layer deposition on $\mathrm{H}$-passivated $\mathrm{Si}(111)$

J. Appl. Phys. 112, 113517 (2012)

Optical response in subnanometer gaps due to nonlocal response and quantum tunneling Appl. Phys. Lett. 101, 233111 (2012)

A two-dimensional nanopatterned thin metallic transparent conductor with high transparency from the ultraviolet to the infrared

Appl. Phys. Lett. 101, 181112 (2012)

Experimental investigation of photonic band gap influence on enhancement of Raman-scattering in metaldielectric colloidal crystals

J. Appl. Phys. 112, 084303 (2012)

\section{Additional information on J. Appl. Phys.}

Journal Homepage: http://jap.aip.org/

Journal Information: http://jap.aip.org/about/about_the_journal

Top downloads: http://jap.aip.org/features/most_downloaded

Information for Authors: http://jap.aip.org/authors

\section{ADVERTISEMENT}

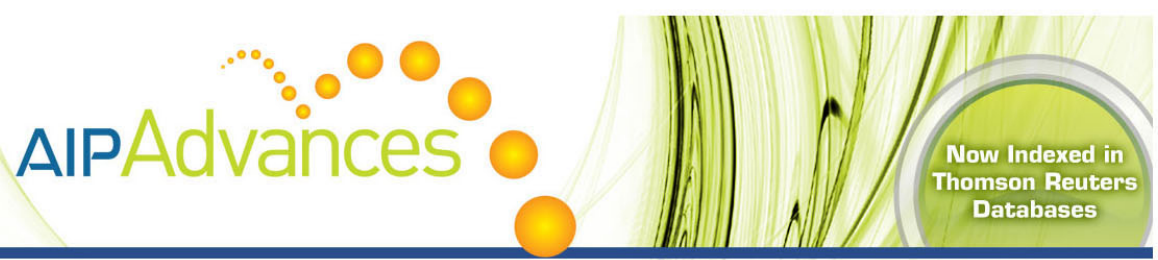

\section{Explore AIP's open access journal: - Rapid publication \\ - Article-level metrics \\ Post-publication rating and commenting}




\title{
Determination of optical properties of percolated nanostructures using an optical resonator system
}

\author{
J. Sukmanowski, ${ }^{1}$ Y. Battie, ${ }^{2, a)}$ F. X. Royer, ${ }^{2}$ and A. En Naciri ${ }^{2}$ \\ ${ }^{1}$ INBITEC e.V. Institut für biologisch-medizinische Forschung und Technologie e.V. Forschungscampus \\ des Max-Delbrück-Centrums für Molekulare Medizin (MDC), Am Sandhaus 31, 13125 Berlin, Germany \\ ${ }^{2}$ LCP-A2MC, Institut Jean Barriol, Université de Lorraine, 1 Bd Arago, 57070 Metz, France
}

(Received 25 July 2012; accepted 31 October 2012; published online 30 November 2012)

\begin{abstract}
In this work, methods are introduced to the determination of optical properties of thin silver films and nanostructures. We present an optical resonant system consisting of a mirror, a transparent layer and a thin silver film. The layer sequences and the nanostructure of the thin films are investigated by optical methods consist of reflectance measurements. The structures are analyzed by atomic force microscopy and scanning electron microscopy. The optical properties are determined by modeling the reflectance data. We have found that the growth mechanisms of the silver layer are correlated to its optical properties. It also found that temperature treatments produce isolated particles with a narrow plasmon resonance. (C) 2012 American Institute of Physics. [http://dx.doi.org/10.1063/1.4768202]
\end{abstract}

\section{INTRODUCTION}

Optical properties of thin metal films may provide an important clue about their internal properties; ${ }^{1-4}$ yet they are difficult to analyze and the determination of their properties remains relevant. In this paper, we study nanostructured thin layers of silver deposited on top of an optically resonant layer system (ORL). ${ }^{5}$ By independently measuring the morphology of the layer, we investigate its effect on the optical spectra. In particular, under the condition that certain geometrical patterns are formed on the surface and the percolation over metallic particles is set up, the real part of the dielectric function gets a negative value. As a consequence, this film can serve as a building block for the development of new metamaterial devices. ${ }^{6}$

The ORL consists of a transparent layer $\left(\mathrm{SiO}_{2}\right.$ or $\left.\mathrm{ZnO}\right)$, of approximately $100 \mathrm{~nm}$ thickness, which roughly corresponds to one fourth of the wavelength, coated on a metallic Ag mirror. ${ }^{7}$ On top of the transparent layer, the silver layers to be studied are deposited; their thickness varied from 1 to $90 \mathrm{~nm}$ (see Fig. 1). In this optical system, the incident light passes through the thin layer and the transparent layer to be reflected by the mirror. Because of the $\lambda / 4$ condition, a constructive interference of the field strength occurs in the thin top layer. This interference increases the absorption of the thin metallic top layer.

The topography of the metallic top layer could be influenced by a post-annealing treatment. ${ }^{8,9}$

The surfaces are examined by atomic force microscopy (AFM) and scanning electron microscopy (SEM). The optical properties were investigated by reflectance measurements. The optical properties of metallic bulk material were already studied in the literature in detail. ${ }^{10}$ In this work, we demonstrate that thin metallic layers on the nanoscale range shows completely different reflection properties than bulk materials. Some discussions are already given. ${ }^{11-13}$ Three questions

\footnotetext{
a) Author to whom correspondence should be addressed. Electronic mail: yann.battie@univ-lorraine.fr.
}

seem to be interesting, (1) which is the critical layer thickness for the change to the optical properties? (2) What is the impact of the topography? (3) How the ORL influences the reflectance spectra.

The answers to the questions should be given to the following approach: (1) The structures will be integrated into an optical resonant structure; therefore small structural changes provide significant measurement signals. (2) The topography will be measured. The change in the topography will be compared to the change in the reflection. (3) The optical system will be fit by standard routines to get the optical properties.

\section{MATERIAL AND METHODS}

\section{A. Sample preparation}

The metallic layer, the metallic nanostructures, the $\mathrm{SiO}_{2}$ layers, and the Ag mirror were produced by thermal evaporation in an UV chamber at a pressure of $10^{-6}$ mbar. The $\mathrm{SiO}_{2}$ layers were thermal treated after deposing at $450^{\circ} \mathrm{C}$ for $10 \mathrm{~h}$ in a furnace at a pressure of approximately $10^{-5}$ mbar. Tests with acetone have shown that the $\mathrm{SiO}_{2}$ layers were chemically stable and ellipsometric measurements revealed that the layers were completely oxidized. The refractive index of wellknown $\mathrm{SiO}_{2}$, i.e., the same as native oxide on silicon wafer, was obtained by ellipsometry but not given here. The thickness of the mirrors was $300 \mathrm{~nm}$. The distance between evaporation sources and sample was $500 \mathrm{~mm}$ for the fabrication of the $\mathrm{SiO}_{2}$ layer. For the production of the metallic nanostructures, the metallic layers and the mirror distance was $350 \mathrm{~mm}$. The support of the mirrors was a plate of standard laboratory glass or polycrystalline silicon. Mixture of different nanoparticle materials in the course of the manufacturing process was prevented by carefully cleaning the vacuum chamber prior to evaporation of a new metal. For test reasons some nanostructures layers were also made by sputtering. The nominal thicknesses were measured by quartz oscillator. The $\mathrm{ZnO}$ layers were made at the Research Center Jülich as described in previous publications. ${ }^{14,15}$ 


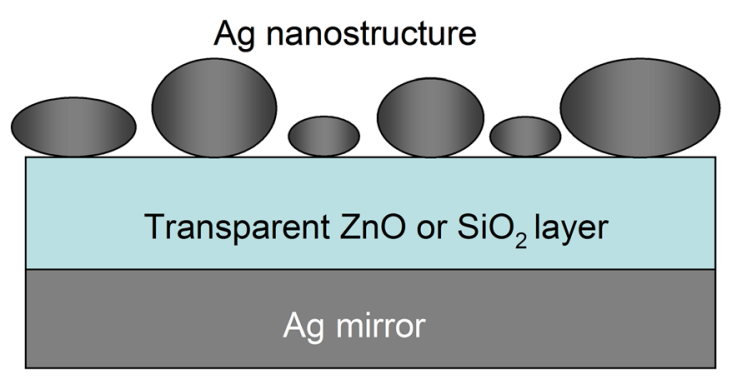

FIG. 1. Schematic presentation of the layer system.

\section{B. Optical and structural measurements}

The reflectance measurements were carried out close to backscattering geometry on a reflectometer at incidence angle of $8^{\circ}$ in the $190 \mathrm{~nm}-1100 \mathrm{~nm}$ wavelength range. The scattered light was also measured in an integral sphere (Ulbricht sphere) to control our measurements and to analyze the diffuse light. ${ }^{16}$

The separated nanoparticles and the metallic layers were analyzed by AFM (nanoscope Nano $\mathrm{R}^{2}$ Pacific nanotechnology) and by a scanning electron microscope. Since $\mathrm{SiO}_{2}$ is an insulator, SEM images are only recorded from Ag deposited on $\mathrm{ZnO}$ ORL or on a c-Si substrate with a naturally thin $\mathrm{SiO}_{2}$ layer.

\section{RESULTS}

\section{A. Reflectance measurements on thin metal layers}

The influence of the nominal layer thickness on the optical properties can be recognized clearly in the reflection measurement as can be seen in Figs. 2(a) and 2(b). Fig. 2(a) shows the results from Ag layer of $17 \mathrm{~nm}, 33 \mathrm{~nm}, 50 \mathrm{~nm}$, and $90 \mathrm{~nm}$ nominal thicknesses applied on the ORL, consisted of a silver mirror and a transparent layer of $100 \mathrm{~nm} \mathrm{ZnO}$. With a $90 \mathrm{~nm}$ nominal silver thickness, the influence of the ORL is barely visible. Fig. 2(b) shows the results from Ag layer of $1 \mathrm{~nm}, 2 \mathrm{~nm}, 3 \mathrm{~nm}, 4 \mathrm{~nm}, 5 \mathrm{~nm}$, and $6 \mathrm{~nm}$ nominal thicknesses applied on the ORL.

For wavelengths shorter than $400 \mathrm{~nm}$, the reflection decreases dramatically for all samples because of internal properties of $\mathrm{ZnO}$ and the interband threshold of silver. The thinnest layer, of $1 \mathrm{~nm} \mathrm{Ag}$, exhibits the reflectance almost indistinguishable from that of the underlying $100 \mathrm{~nm}$ thick $\mathrm{ZnO}$ without $\mathrm{Ag}$; only that around $570 \mathrm{~nm}$ a slight dip in the

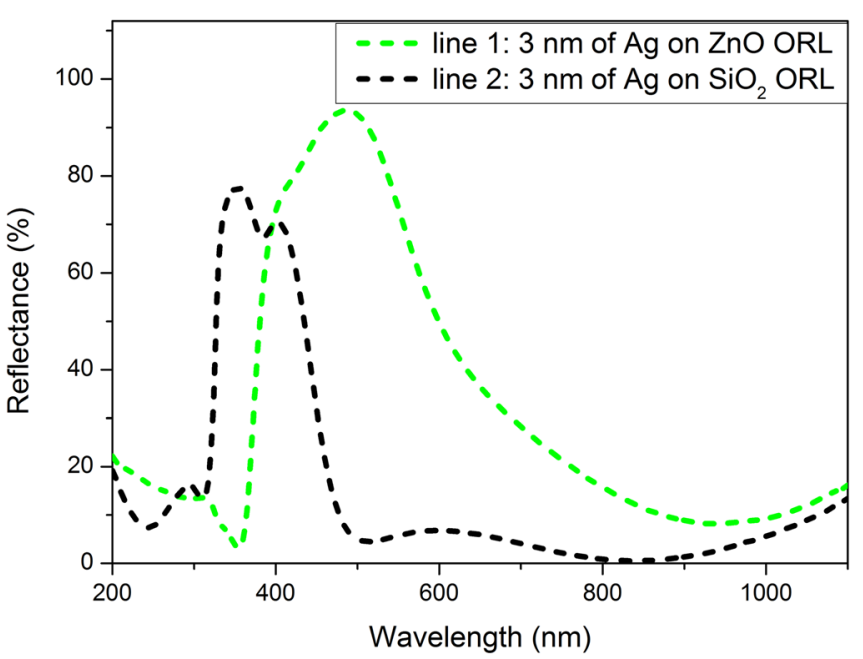

FIG. 3. Experimental reflectance spectra obtained from Ag layer of $3 \mathrm{~nm}$ nominal thicknesses applied on the $\mathrm{ZnO}$ and $\mathrm{SiO}_{2}$ ORLs.

reflectance is visible. On the contrary, Figs. 2(a) and 2(b) show that thicker Ag layers exhibit large change. For a $4 \mathrm{~nm}$ layer, the reflectance drops to almost zero at $800 \mathrm{~nm}$ wavelengths. Obviously, a certain change in behavior happens between $4 \mathrm{~nm}$ (Fig. 2(b)) and $17 \mathrm{~nm}$ (Fig. 2(a)) layer nominal thickness. For a $17 \mathrm{~nm}$-thick Ag layer, a pronounced minimum in reflectance occurs at around $600 \mathrm{~nm}$ wavelengths. This minimum becomes sharper, deeper, and shifts to shorter wavelength for $33 \mathrm{~nm}$-thick Ag layer. It persists also for thicker layers but it becomes ever less deep as the nominal layer thickness increases, until it ultimately disappears for $90 \mathrm{~nm}$. This minimum provides from the destructive interference between light waves reflected by the upper and lower ORL boundaries. In other words, the ORL is narrow notch filter structure which filters a narrow spectral range.

\section{B. Comparison between reflectance measurements on $\mathrm{SiO}_{2}$ and $\mathrm{ZnO}$ ORL}

Fig. 3 shows comparison between the experimental reflectance spectra of $3 \mathrm{~nm} \mathrm{Ag}$ layers deposited on a $\mathrm{ZnO}$ ORL and on a $\mathrm{SiO}_{2}$ ORL. Both spectra have similar features such as a minimum and a maximum in reflectance in the $800-1000 \mathrm{~nm}$ and $350-500 \mathrm{~nm}$ wavelength ranges, respectively, providing from the destructive and the constructive interference between the incident and the reflected light on the ORL. However, due to the lower refractive index of $\mathrm{SiO}_{2}$ than

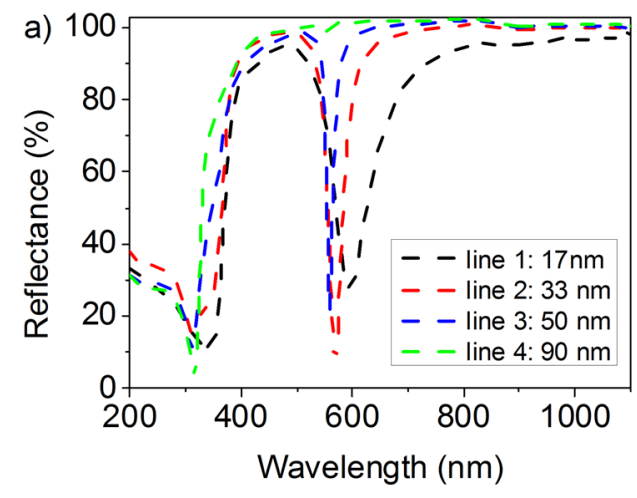

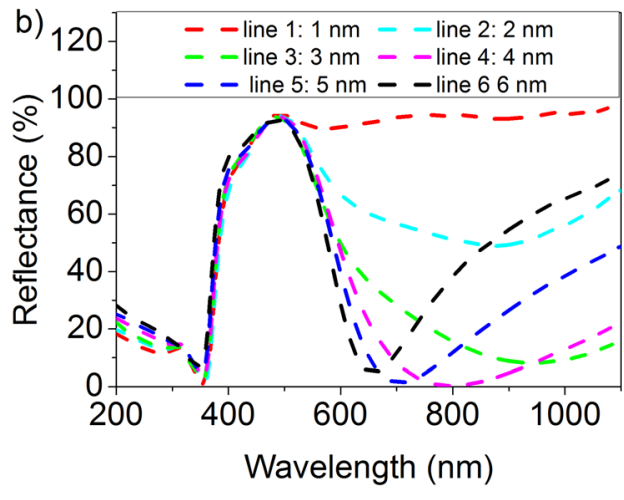

FIG. 2. Experimental reflectance spectra obtained from $\mathrm{Ag}$ layer of (a) $17 \mathrm{~nm}$, $33 \mathrm{~nm}, 50 \mathrm{~nm}$, and $90 \mathrm{~nm}$ and (b) $1 \mathrm{~nm}$, $2 \mathrm{~nm}, 3 \mathrm{~nm}, 4 \mathrm{~nm}, 5 \mathrm{~nm}$, and $6 \mathrm{~nm}$ nominal thicknesses applied on the ZnO ORL. 


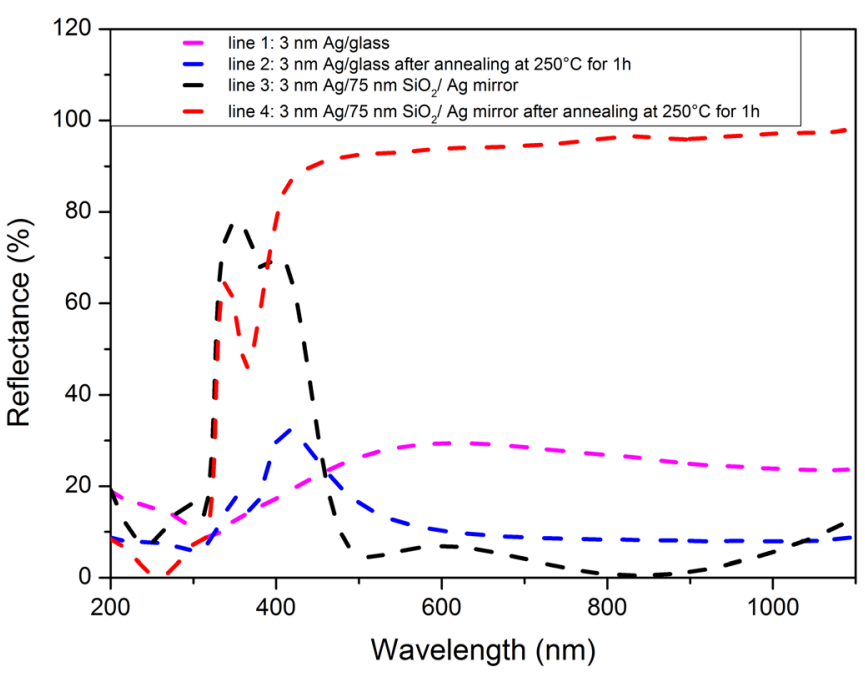

FIG. 4. Experimental reflectance spectra of silver layers on $\mathrm{SiO}_{2}$ substrates (lines 1 and 2) or $\mathrm{a} \mathrm{SiO}_{2}$ ORL (lines 3 and 4) before (lines 1 and 3 ) and after (lines 2 and 4) annealing.

$\mathrm{ZnO}$, the minimum and the maximum in reflectance of the $\mathrm{Ag}$ layer on the $\mathrm{SiO}_{2}$ ORL are both blueshifted by nearly $120 \mathrm{~nm}$.

A slight dip in reflectance at $380 \mathrm{~nm}$, attributed to the plasmon resonance of silver nanoparticles is also observed for the $\mathrm{SiO}_{2}$ ORL. This feature is not present on the $\mathrm{ZnO}$ ORL reflectance spectra probably due to a substrate dependence of the silver island shape and nucleation rate.

\section{Reflectance measurements before and after annealing}

We turn now to the discussion of annealing effects on the optical properties of metal layers. Two systems have been taken for such an analysis: (i) a $3 \mathrm{~nm}$ Ag layer on a laboratory glass and (ii) the same Ag layer, but deposited on 75 nm-thick $\mathrm{SiO}_{2}$ layer and a thick $\mathrm{Ag}$ mirror behind it (all this mounted on a laboratory glass as well). The reflectance spectra of both systems before and after annealing at $250{ }^{\circ} \mathrm{C}$ during $1 \mathrm{~h}$ are shown in Fig. 4. As shown in Fig. 4, the reflectance dispersion curves of the second systems present higher variations. The ORL structure improves the sensitivity to both, the morphology and the optical properties of the Ag film. The system after annealing (line 4) shows a stronger dip in reflectance at $380 \mathrm{~nm}$ than unannealed system. It has the same reflectivity spectrum in the range from 400 to $1100 \mathrm{~nm}$ as the same system without deposited upper layer (spectrum not shown), whereas for the freshly deposited Ag layer of system, the reflectivity is very different (line 3 ). Please note that the topography of the nanostructures is changed by heating (see Sec. III D) and that the structures are investigated by a resonant system, which works very well for line 3 .

It is worth to note that the $\mathrm{SiO}_{2}$ layers are very stable and contains negligible levels of impurities; ${ }^{14,15}$ therefore we can exclude the diffusion of silver into the $\mathrm{SiO}_{2}$. ${ }^{17,18}$

The reflectance decreased to zero, in all cases if we used a $3 \mathrm{~nm}$ evaporated silver layer on top of a transparent layer $\left(\mathrm{ZnO}\right.$ or $\left.\mathrm{SiO}_{2}\right)$ coated on $\mathrm{Ag}$ mirror.

\section{Topographic measurements}

Concerning the discussion of layer topography, it is shown in Fig. 5 the SEM images of $3 \mathrm{~nm}, 10 \mathrm{~nm}$, and $20 \mathrm{~nm}-$ thick silver layers on $\mathrm{ZnO}$ ORL.

The $3 \mathrm{~nm}$-thick silver layer is composed of nearly percolated silver islands with complex elongated shape and randomly orientations (Fig. 5(a)). SEM image of a $10 \mathrm{~nm} \mathrm{Ag}$ layer (Fig. 5(b)) reveals that the Ag islands tend to percolate, forming a continuous film. Above a $20 \mathrm{~nm}$ thickness, silver completely covers the $\mathrm{ZnO}$ surface (Fig. 5(c)).

Figs. 6(a) and 6(b) shows the SEM images of $10 \mathrm{~nm}$ nominal thick silver on top of c-Si and Figs.6(c) and 6(d) shows a $3 \mathrm{~nm}$ nominal thick $\mathrm{Ag}$ on top of $\mathrm{ZnO}$, each before and after annealing. ${ }^{14}$ On the c-Si substrate exists a naturally $\mathrm{SiO}_{2}$ layer, which has the same refractive index as the ORL $\mathrm{SiO}_{2}$ layer (ellipsometric measurements are not shown). Therefore, we can expect similar nanostructures on c-Si as on laboratory glass of $\mathrm{SiO}_{2}$ or on evaporated $\mathrm{SiO}_{2}$ layers.

Considering a $10 \mathrm{~nm}$ thick Ag layer, silver islands on $\mathrm{SiO}_{2}$ (Fig. 5(b)) are smaller and less percolated than on $\mathrm{ZnO}$ ORL (Fig. 6(a)). This is in agreement with the slight dip in reflectance observed at $380 \mathrm{~nm}$ in Fig. 3 (line 2) attributed to the plasmon resonance of some isolated silver islands. In other words, the layer on the $\mathrm{SiO}_{2}$ ORL consists in an intermediate system between isolated and percolated silver islands. The shape of silver islands depends on the ability of adatoms to diffuse on the substrate surface suggesting a substrate nucleation rate dependance.

Comparing to the SEM images of unannealed samples (Figs. 6(a) and 6(c)), silver islands become less elongated and their average size increases after the annealing step
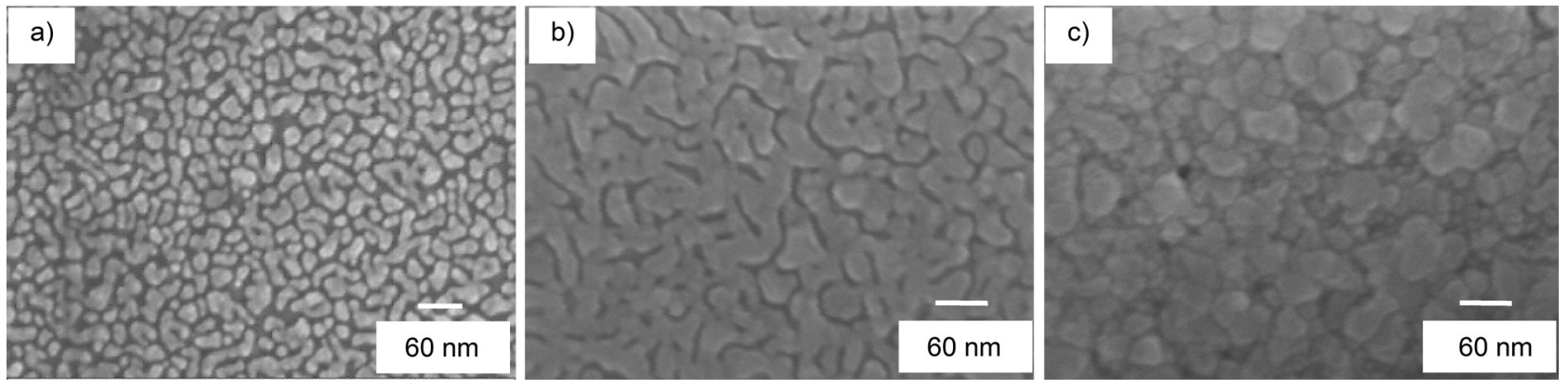

FIG. 5. (a) SEM images of a $3 \mathrm{~nm}$, (b) $10 \mathrm{~nm}$, and (c) $20 \mathrm{~nm}$-thick Ag layer on a $\mathrm{ZnO}$ ORL. 

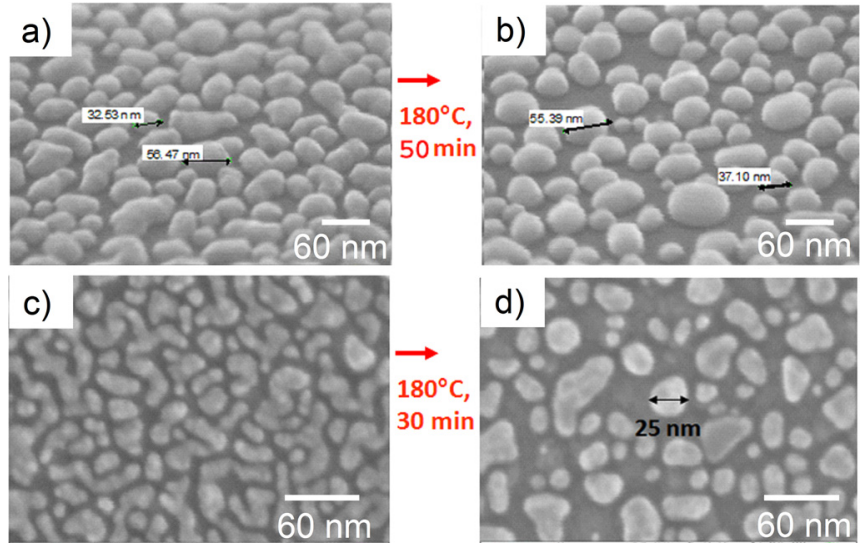

FIG. 6. SEM images of metallic island films obtained by thermal evaporation of $10 \mathrm{~nm} \mathrm{Ag}$ on c-Si before (a) and after (b) annealing treatment at $180^{\circ} \mathrm{C}$ during $50 \mathrm{~min}$. SEM images of metallic island films obtained by thermal evaporation of $3 \mathrm{~nm} \mathrm{Ag}$ on $\mathrm{ZnO}$ before (c) and after (d) annealing treatment at $180^{\circ} \mathrm{C}$ during $30 \mathrm{~min}$.

(Figs. 6(b) and 6(d)). For a $3 \mathrm{~nm}$ thick film, the surface density of $\mathrm{Ag}$ islands is also reduced from $62 \%$ to $38 \%$ on $\mathrm{ZnO}$ ORL. Thus, the annealing generates more isolated silver nanoparticles. The formation of these isolated silver nanoparticles is attributed to different thermal expansion coefficients between $\mathrm{Ag}$ islands and the transparent layer and to the relaxation of the thermal stress during the annealing. ${ }^{8,9}$

An AFM image of the same Ag layer on a glass after annealing is shown in Fig. 7. It is important that layer consists of well pronounced isolated nanoparticles even after annealing, showing a tendency of sintering. Namely, their measurements indicate that the Ag surface is smooth and the thickness is higher than the nominal thickness. Contrary to AFM measurement, the microbalance underestimates the thickness since the film is not homogeneous but composed of isolated $\mathrm{Ag}$ islands. This means the film density is lower than the bulk silver density taken to determine the nominal thickness.

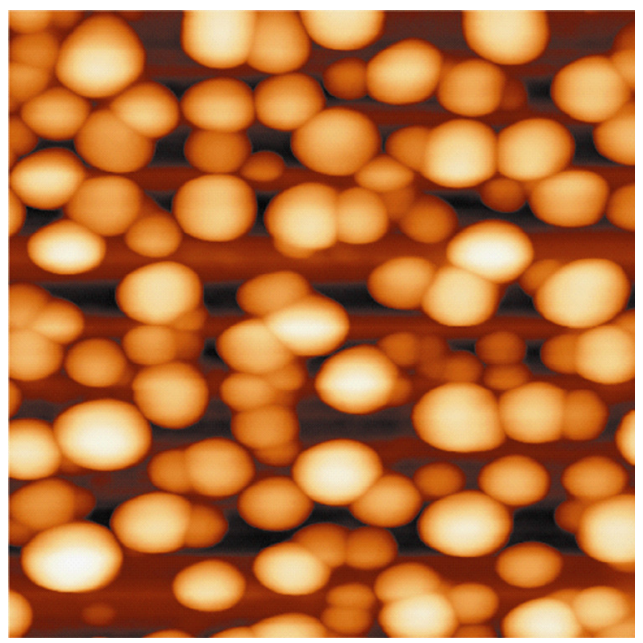

FIG. 7. AFM image of $3 \mathrm{~nm} \mathrm{Ag}$ on laboratory glass on an area of $1 \mu \mathrm{m}$ $\times 1 \mu \mathrm{m}$ after annealing $\left(250^{\circ} \mathrm{C}\right.$ for $\left.1 \mathrm{~h}\right)$. The nanoparticles are separated. The nanoparticles have an average height and diameter estimated at $30 \mathrm{~nm}$ and $120 \mathrm{~nm}$, respectively.

\section{DISCUSSION}

The measured reflectance spectra compared to the theoretical ones are also performed. The theoretical model is based on the calculation of reflectance from a model which consists of three layers: the $\mathrm{Ag}$ mirror, the $\mathrm{ZnO}$ or the $\mathrm{SiO}_{2}$ layer and top layer composed of a mixture of $\mathrm{Ag}$ and void. Effective medium approximation determines the effective dielectric functions of a composite medium as a mathematical combination of the dielectric functions of its constituent. Maxwell Garnett (MG) ${ }^{19}$ and Bruggeman effective medium approximation (BMA) ${ }^{20}$ formulated, respectively, by below equations,

$$
\begin{gathered}
\frac{\varepsilon_{e f f}(\lambda)-1}{\varepsilon_{e f f}(\lambda)+2}=f \frac{\varepsilon_{A g}(\lambda)-1}{\varepsilon_{A g}(\lambda)+2}, \\
0=(1-f) \frac{1-\varepsilon_{e f f}(\lambda)}{1+2 \varepsilon_{e f f}(\lambda)}+f \frac{\varepsilon_{A g}(\lambda)-\varepsilon_{e f f}(\lambda)}{\varepsilon_{A g}(\lambda)+2 \varepsilon_{e f f}(\lambda)},
\end{gathered}
$$

are used and compared. Where $\varepsilon_{\mathrm{Ag}}$ is the dielectric function of $\mathrm{Ag}$ and $\varepsilon_{\mathrm{eff}}$ is the effective dielectric function of the medium composed of a mixture of silver and void, $f$ the volume fraction of Ag and $\lambda$ the wavelength. MG theory gives a realistic description of a medium when the volume fraction of inclusions, weakly interacting with each other, is smaller than that of the host material. BMA describes the optical properties of composite mediums where inclusions and host material have similar roles in the effective medium.

Considering the size of $\mathrm{Ag}$ islands, surface damping effect can be neglected. ${ }^{21}$ The Ag dielectric function was taken from the literature as for bulk silver. ${ }^{10}$ The $\mathrm{ZnO}$ and $\mathrm{SiO}_{2}$ dielectric function was also extracted from the literature without any additional fitting. Only three parameters are fitted: the $\mathrm{ZnO}$ or $\mathrm{SiO}_{2}$ film thickness, the $\mathrm{Ag}$ film thickness, and the volume fraction $\mathrm{f}$ of $\mathrm{Ag}$. Some examples of such comparison are given in Fig. 8. Close to the UV range $(200 \mathrm{~nm}$ to $300 \mathrm{~nm})$, the measured and calculated spectra show only a trend line. In this spectral region where the reflectance is essentially attributed to both, the interband transitions of $\mathrm{Ag}$ and the $\mathrm{ZnO}$ layer, further detailed studies are necessary. However, from $300 \mathrm{~nm}$, the resulting curve is in fair agreement with the measured one. It was not possible to find a serious fit of the reflectance without the ORL for the lines 1 and 2 in Fig. 4. Contrary to these spectra, the reflectance for lines 3 and 4 provides from the multi absorption and multi reflection in the ORL. This suggests that the ORL structure improves the sensitivity to the optical properties of the top layer.

For the $1 \mathrm{~nm}$ nominal thick $\mathrm{Ag}$ layer, $\mathrm{MG}$ and BMA (not shown) can both be used to model the reflectance spectra indicating a threshold condition between both models. However, BMA gives a slightly better fit. Above $1 \mathrm{~nm}, \mathrm{MG}$ effective medium approximation fails to predict the reflectance spectra of the as-deposited Ag film while the calculated spectrum from BMA is in agreement with the measured one (Fig. 8). MG works well for isolated particles embedded in a host matrix. As shown in Fig. 5(a) for a $3 \mathrm{~nm}$ thick Ag layer, both compound, i.e., Ag and void, plays a symmetric rule, 


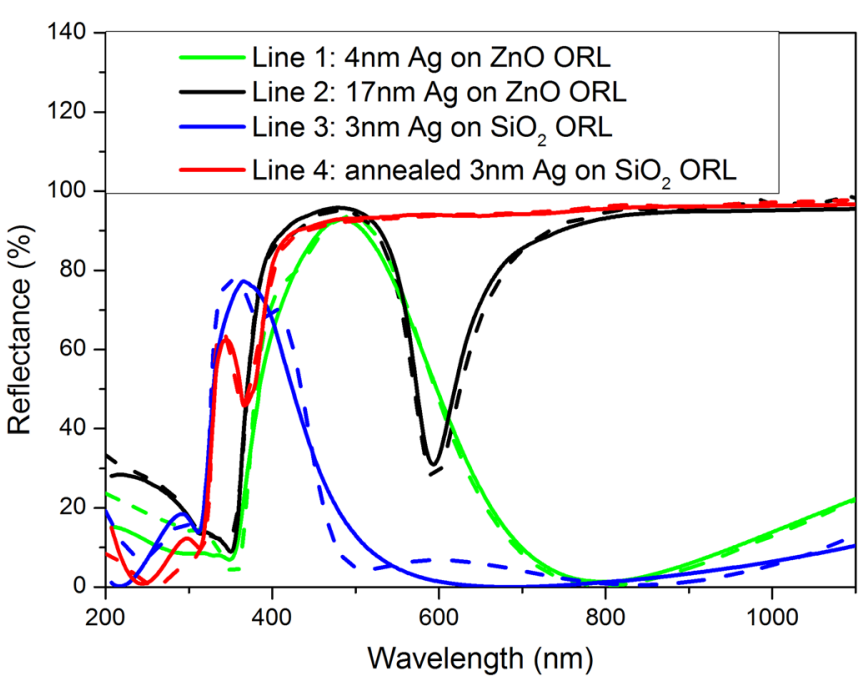

FIG. 8. Reflectance measurements (dash lines) and calculated spectra (solid lines) for 4, $17 \mathrm{~nm}$-thick $\mathrm{Ag}$ layers on $\mathrm{ZnO}$ ORLs and $3 \mathrm{~nm}$-thick Ag layers on $\mathrm{SiO}_{2}$ ORLs before and after an annealing treatment.

suggesting that the reflectivity spectra must be interpreted in term of BMA (Fig. 8). Above $17 \mathrm{~nm}$, the experimental data can be analysed thanks to a simple homogeneous silver film without void. As expected, the Ag islands tend to percolate (Fig. 5(b)), forming a continuous film (Fig. 5(c)).

As shown in Fig. 9, the thickness of the Ag layer on the $\mathrm{ZnO}$ ORL, deduced from reflectance measurement, is higher than the nominal thickness but is in agreement with AFM measurements (Fig. 7). As we introduced previously, microbalance measurements underestimate the film thickness. We will discuss now the dynamics of nucleation, percolation and the early stages of bulk Ag film. In the initial stages of film formation, the thickness measured by reflectivity and the volume fraction of silver both increase rapidly. Beyond a $4 \mathrm{~nm}$ nominal thickness, the thickness slightly decreases and remains constant in the nominal thickness range of $6 \mathrm{~nm}-$ $17 \mathrm{~nm}$. This behaviour was observed by Little et al., ${ }^{22}$ and indicates that the film grows in a Volmer-Weber mode. ${ }^{23-25}$

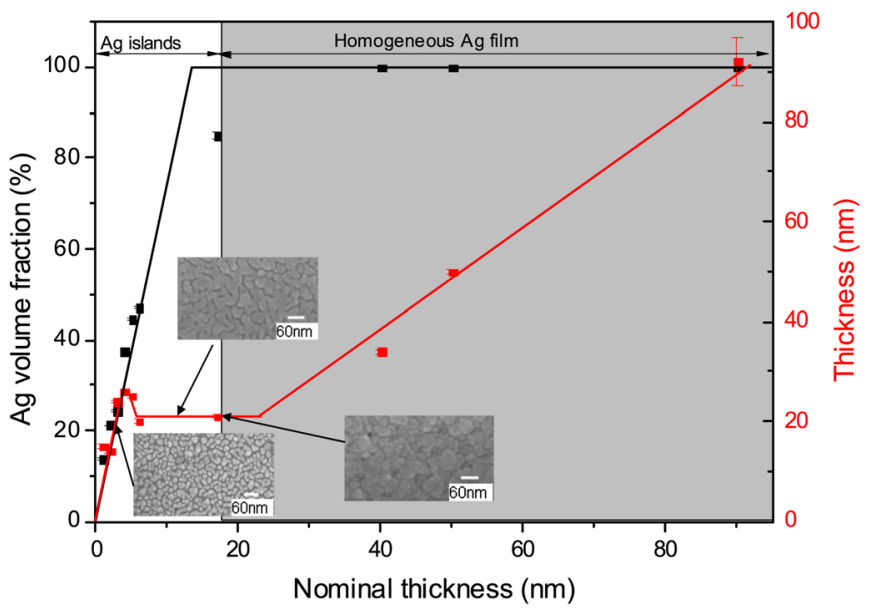

FIG. 9. Ag volume fraction and thickness of the deposited layer on the $\mathrm{ZnO}$ ORL, calculated by fitting the experimental reflectance spectra, versus the nominal thickness estimated from mass measurements. The dots are the results of the fits. SEM images of a $3 \mathrm{~nm}$, a $10 \mathrm{~nm}$, and a $20 \mathrm{~nm}$-thick Ag layer on a $\mathrm{ZnO}$ ORL are included to facilitate the analysis.
Adatoms migrate on the surface until they nucleate on other adatoms resulting in the formation of $\mathrm{Ag}$ islands (Fig. 5(a)). Further deposition fills empty space between the islands (Fig. 5(b)). At this step, the volume fraction of the film increases without any modifications of the film thickness until the islands begin to agglomerate and form a percolated network. ${ }^{23}$ Once the surface is completely covered, the film thickness starts to increase linearly with the nominal thickness suggesting that the film grows like a layer by layer in a Frank-Van der Merwe mode. ${ }^{24,26}$ Finally, a homogeneous $\mathrm{Ag}$ layer is formed (Fig. 5(c)).

The real part of the effective dielectric function (Fig. 10(a)) decreases monotonously when the nominal film thickness increases. When the nominal thickness becomes higher than $4 \mathrm{~nm}$, the real part of the effective dielectric function takes negative value in the visible spectral range. As reported by Oates et al., ${ }^{26}$ the percolation threshold can be determined from the effective dielectric function of the Ag layer. It corresponds to the nominal thickness where the real part of the effective dielectric function falls below zero. In agreement with SEM investigations (Figs. 5(a) and 5(b)), for this nominal thickness estimated between $4 \mathrm{~nm}$ and $6 \mathrm{~nm}$, the silver volume fraction varies between $38 \%$ and $48 \%$, and the film transits from a dielectric to a conductor. The imaginary part of the effective dielectric function (Fig. 10(b)) of the $1 \mathrm{~nm}$ Ag layer has a wide band centred at $500 \mathrm{~nm}$ which is attributed to the plasmon resonance of the composite layer. ${ }^{27}$ Due to the electro-magnetic interactions between islands, this band is redshifted and becomes progressively wider as the film grows. ${ }^{27}$ The imaginary part of the effective dielectric function of a $4 \mathrm{~nm}$ thick $\mathrm{Ag}$ film is larger than the $\mathrm{Ag}$ bulk one. This high absorption induces that the refection goes to the zero line in the visible range. Beyond a $4 \mathrm{~nm}$ film thickness, the opposite trend is observed. The plasmon band is blueshifted and becomes progressively narrower as the film grows. Finally, for high nominal film thickness, the dielectric function tends toward the Ag bulk one.

Before annealing, the optical properties of the silver composite layer deposited on the $\mathrm{SiO}_{2}$ ORL are nearly described by the BMA approximation (Fig. 8, line 3). Some deviations between the experimental data and the calculated ones, attributed to the presence of isolated islands in the nearly percolated network (Fig. 6(a)), are observed. It suggests that other effective medium models need to be developed to describe the optical properties of silver layer in the intermediate state between percolated and nonpercolated silver islands. The post-annealing process alters significantly the structural properties (Figs. 6(b) and 6(d)) and the optical properties (Fig. 4) of the film. ${ }^{8}$ It produces isolated Ag nanoparticles with nearly spherical shape (Figs. 6(b), 6(d), and 7). After the annealing, important deviation between BMA and the experimental data are observed (not shown). As shown in Figs. 6(b)-6(d)) and Fig. 7, the concentration of silver islands is sufficiently low to consider them as completely isolated inclusion. Indeed, for a $3 \mathrm{~nm}$ thick film, the surface density of $\mathrm{Ag}$ islands is reduced from $62 \%$ to $38 \%$. Thus, MG approximation well describes the optical properties of the annealed Ag layer (Fig. 8). The imaginary part of the effective dielectric function of the $\mathrm{Ag}$ layer presents a 

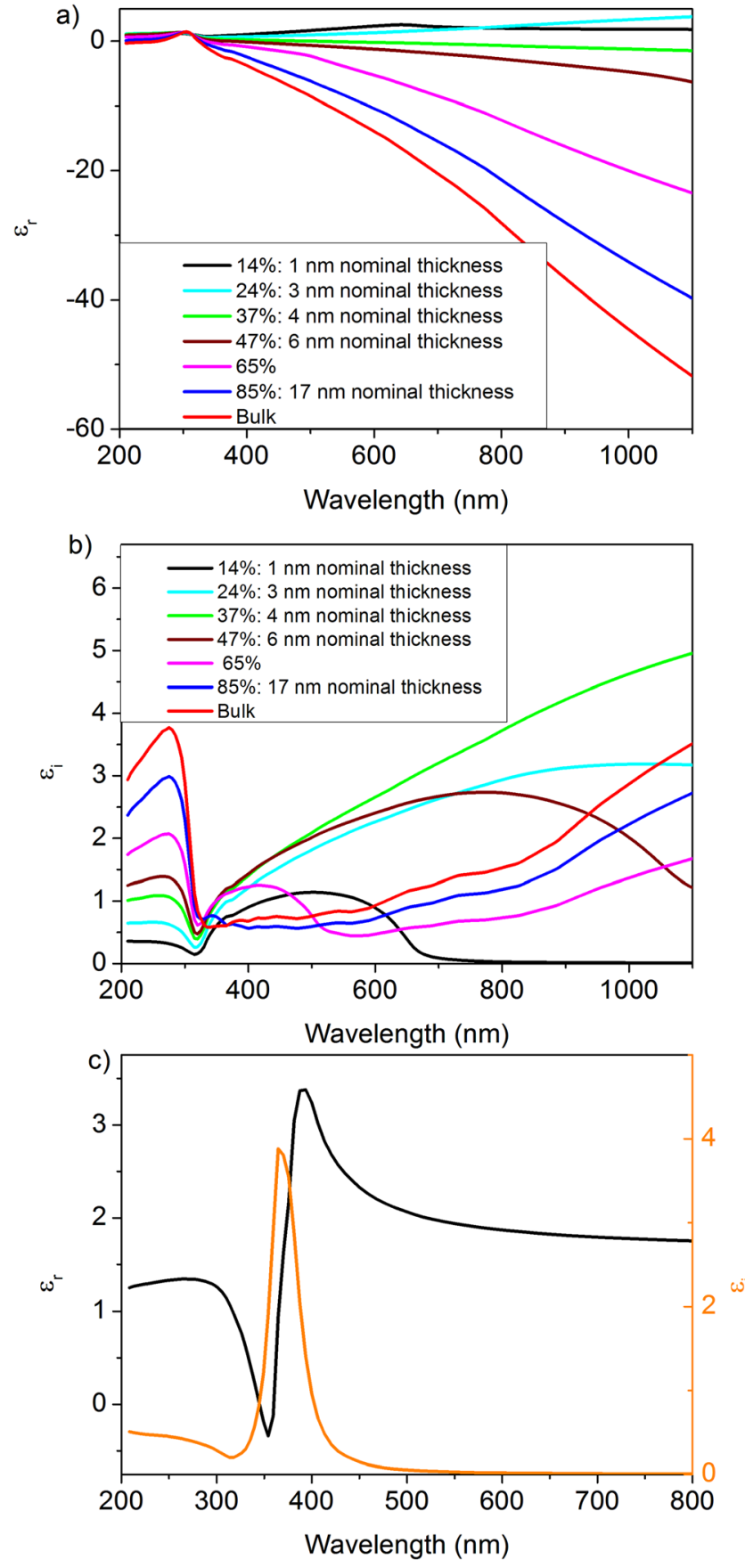

FIG. 10. Variations of the real part (a) and imaginary part (b) of the effective dielectric function of the $\mathrm{Ag}$ layer during the deposition on a $\mathrm{ZnO}$ ORL. The curves calculated for $65 \%$ of $\mathrm{Ag}$ volume fraction is added to facilitate the interpretation. (c) Real part and imaginary part of the effective dielectric function of the $3 \mathrm{~nm}$ thick $\mathrm{Ag}$ layer on $\mathrm{SiO}_{2}$ ORL after the annealing.

narrow band centred at $380 \mathrm{~nm}$ (Fig. 10(c)). This band is the plasmon resonance of the isolated metallic islands. ${ }^{4,28}$ This absorption induces a decrease in reflectivity at $380 \mathrm{~nm}$ (Fig. 8). In accordance with Kramers-Kronig relations, ${ }^{29}$ the real part of the effective dielectric function of the Ag layer varies strongly close to the plasmon resonance wavelength. As the complex dielectric function is weakly disturbed beyond this wavelength, the reflectivity in the visible spectral range is similar to that of the structure before the Ag deposition (not shown).
Thus, the effective dielectric function can be tuned by varying the nominal thickness or by using a post-annealing process. Its real part can take a negative value which is suitable for designing new metamaterial devices. ${ }^{27,30,31}$

\section{CONCLUSION}

This work shows that reflectometry on the ORL is a straightforward measurement to study the percolation of thin silver films and their modification by annealing. The ORL enhances the sensitivity to the optical properties of the thin top layer. The modeling with the effective medium approximation highlights the growth mechanisms of the silver layer. In the first step it grows in a Volmer-Weber mode and after a complete coverage of the surface, it grows in a Frank-Van der Merwe mode. From the model, the percolation threshold can be estimated to a nominal thickness of nearly 4-6 nm. The annealing of silver layers generates isolated silver particles with a narrow plasmon resonance. The studies have shown that the effective dielectric is negative in the visible range for percolated silver films, which is useful for optical applications.

\section{ACKNOWLEDGMENTS}

The authors knowledge the Saarland University for its contribution.

${ }^{1}$ R. W. Cohen, G. D. Cody, M. D. Coutts, and B. Abeles, Phys. Rev. B 8, 3689 (1973).

${ }^{2}$ R. S. Sennett and G. D. Scott, J. Opt. Soc. Am. 40, 203 (1950).

${ }^{3}$ S. Yoshida, T. Yamaguchi, and A Kinbara, J. Opt. Soc. Am. 61, 62 (1971). ${ }^{4}$ K. L. Kelly, E. Coronado, L. L. Zhao, and G. C. Schatz, J. Phys. Chem. B 107, 668 (2003).

${ }^{5}$ G. A. N. Connell, R. J. Nemanich, and C. C. Tsai, Appl. Phys. Lett. 36, 31 (1980).

${ }^{6}$ D. R. Smith, J. B. Pendry, and M. C. K. Wiltshire, Science 305, 788 (2004).

${ }^{7}$ S. Ducourtieux, V. A. Podolskiy, S. Gresillon, S. Buil, B. Berini, P. Gadenne, A. C. Boccara, J. C. Rivoal, W. A. Bragg, K. Banerjee, V. P. Safonov, V. P. Drachev, Z. C. Ying, A. K. Sarychev, and V. M. Shalaev, Phys. Rev. B 64, 1654031 (2001).

${ }^{8}$ R. S. Moirangthem, M. T. Yaseen, P.-K. Wei, J.-Y. Cheng, and Y.-C. Chang, Biomed. Opt. Express 3, 899 (2012).

${ }^{9}$ S. A. Little, T. Begou, R. W. Collins, and S. Marsillac, Appl. Phys. Lett. 100, 051107 (2012).

${ }^{10}$ E. D. Palik, Handbook of Optical Constants of Solids (Academic Press Handbook Series, New York, 1985).

${ }^{11}$ J. Sukmanowski, J -R.Viguié, B. Nölting, and F.-X. Royer, J. Appl. Phys. 97, 104332 (2005).

${ }^{12}$ A. Leitner, Z. Zhao, H.Brunner, F. R. Aussenegg, and A. Wokaun, Appl. Opt. 32, 102 (1993).

${ }^{13}$ R. R. Singer, A. Leitner, and F. R. Aussenegg, J. Opt. Soc. Am. B 12, 220 (1995).

${ }^{14}$ E. Moulin, J. Sukmanowski, P. Luo, R. Carius, F. X. Royer, and H. Stiebig, J. Non-Cryst. Solids 354, 2488 (2008).

${ }^{15}$ E. Moulin, P. Luo, B. Pieters, J. Sukmanowski, J. Kirchhoff, W. Reetz, T. Müller, R. Carius, F.-X. Royer, and H. Stiebig, Appl. Phys. Lett. 95, 033505 (2009).

${ }^{16}$ J. Sukmanowski, C. Paulick, O. Sohr, and F. X. Royer, J. Appl. Phys. 88, 2484 (2000).

${ }^{17}$ Y. Battie, N. Destouches, F. Chassagneux, D. Jamon, L. Bois, N. Moncoffre, and N. Toulhoat, Opt. Mater. Express 1, 1019 (2011).

${ }^{18}$ W. Li, S. Seal, E. Megan, J. Ramsdell, K. Scammon, G. Lelong, L. Lachal, and K. A. Richardson, J. Appl. Phys. 93, 9553 (2003).

${ }^{19}$ J. C. M. Garnett, Philos. Trans. R. Soc. London, Ser. A 203, 385 (1904).

${ }^{20}$ D. A. G. Bruggeman, Ann. Phys. 416, 665 (1935). 
${ }^{21}$ U. Krebig and M. Volmer, Optical Properties of Metal Clusters (Springer, Berlin, 1995).

${ }^{22}$ S. A. Little, R. W. Collins, and S. Marsillac, Appl. Phys. Lett. 98, 101910 (2011).

${ }^{23}$ T. W. H. Oates, L. Ryves, and M. M. M. Bilek, Opt. Express 16, 2302 (2008).

${ }^{24}$ H. T. Beyene, J. W. Weber, M. A. Verheijen, M. C. M. Van de Sanden, and M. Creatore, Nano Res. 5, 513 (2012).
${ }^{25}$ S. Marsillac, S. A. Little, and R. W. Collins, Thin Solid Films 519, 2936 (2011).

${ }^{26}$ T. W. H. Oates and A. Mücklich, Nanotechnology 16, 2606 (2005).

${ }^{27}$ J. Sancho-Parramon, V. Janicki, and H. Zorc, Opt. Express 18, 26915 (2010).

${ }^{28}$ A. L. Gonzalez and C. Noguez, Phys. Status Solidi C 4, 4118 (2007).

${ }^{29}$ R. de L. Kronig, J. Opt. Soc. Am. 12, 547 (1926).

${ }^{30}$ J. B. Pendry, Phys. Rev. Lett. 85, 3966 (2000).

${ }^{31}$ N. Fang, H. Lee, C. Sun, and X. Zang, Science 308, 534 (2005). 